\title{
The Effects of Scapular Alignment Exercise and Nerve Mobilization on Pain and Muscle Activity in Subjects with Scapular Depression Alignment
}

\author{
Jong-ho Seok, MSc; Tae-ho Kim, Ph.D \\ Department of Physical Therapy, College of Rehabilitation Science, Daegu University, Daegu, South Korea
}

Background Scapular depression syndrome is associated with weakness or elongation in the upper trapezius and a lower pressure pain threshold.

Purpose This study aimed to compare the pressure pain thresholds of the upper trapezius and median nerve trunks and muscle activity during shoulder flexion before and after scapular alignment exercises and nerve mobilization in subjects with scapular depression alignment.

Study design Two-group pre- and post-test design

Methods The subjects were 30 men with scapular depression alignment aged 20-40 years old. Thirty subjects were randomly divided into the nerve mobilization group $(n=15)$ and the scapular alignment exercise group. The scapular alignment exercise group repeated the scapular setting exercise 15 times a session while maintaining $1 \mathrm{~min}$ per trial, and the nerve mobilization group performed five sets of nerve mobilization, with set including 10, 12, 14, 16 and 20 times. The subjects were measured in terms of the pressure pain threshold and muscle activity during shoulder flexion. The pressure pain threshold was measured in the median nerve and upper trapezius. Muscle activity was measured in the upper trapezius, lower trapezius, and serratus anterior muscles during $120^{\circ}$ shoulder flexion.

Results There was a significant increase in the pressure pain threshold of the upper trapezius before and after intervention in the scapular alignment exercise group $(p<0.05)$. A significant increase in the muscle activity of the serratus anterior before and after intervention was observed in the scapular alignment exercise group $(p<0.05)$, and a significant difference was found in the muscle activity of the serratus anterior between the two groups according to the intervention $(p<0.05)$.

Conclusions The scapular alignment exercises enable subjects with scapular depression alignment to reduce the mechanical sensitivity of muscles and nervous tissues and bring immediate activation in muscle activity of serratus anterior. This study recommend that scapular alignment exercise could help scapular depression syndrome as a therapeutic exercise method in clinical field.

Key words Nerve mobilization, Scapular alignment, Scapular depression, Scapular setting.

\section{INTRODUCTION}

Scapular dyskinesis is defined as the position and movement of the scapula relative to the thoracic cage, and it is a general term that reflects the loss of normal control of
JMST

2020; 4(2): 58-65 Published Online Dec 31, 2020

pISSN 2635-8573 eISSN 2635-8581

Article History

Received 14 Oct 2020 Revised 20 Oct 2020 (1st)

Revised 22 Oct 2020

(2nd)

Accepted 26 Oct 2020

\section{CONTACT}

hohoho90@naver.com

Tae-ho Kim,

Department of Physical

Therapy, College of

Rehabilitation Science,

Daegu University, Daegu,

South Korea

This is an Open-Access article distributed under the terms of the Creative Commons Attribution Non-Commercial License (http://creativecommons org/licenses/by-nc/4.0) which permits unrestricted non-commercial use, distribution, and reproduction in any medium, provided the original work is properly cited. 
Scapular depression syndrome is associated with weakness or elongation in the upper trapezius ${ }^{4}$ and a low pressure pain threshold. ${ }^{5}$ Lee et al. found that the scapular depression alignment group had a lower pressure pain threshold in the upper and middle trapezius, and that the muscle activity of the upper trapezius was delayed during the shrug movement. ${ }^{6}$ The decrease in the pressure pain threshold reflects an increase in muscle tissue sensitivity, which is a response to the persistent strain of the upper trapezius held in an excessively elongated position with shoulder depression alignment ${ }^{5}$. Owing to this posture, the elongated and suppressed gastric triceps and scapulae cause cervical stiffness, cervical myofascial tenderness, and pain. ${ }^{7}$ Scapular depression alignment increases the tension of the C5 and C6 vertebral nerves, the lateral cord of brachial plexus, ${ }^{8}$ and the median nerve in the forearm. ${ }^{9}$ Elevation of the shoulder girdle in this alignment can alleviate these stressors and potentially lead to "decompressing" the thoracic outlet. ${ }^{10}$

The muscles connected to the scapula and the neck in the scapular depression alignment continuously pull the neck downward, increasing the pain sensitivity above the joint between the cervical processes..$^{5}$ The scapular depression alignment can cause prolonged and repetitive stress and pressure in the brachial plexus, sensitize nervous tissue, and cause neck-arm pain symptoms. ${ }^{4}$ The neurodynamic test and the pressure pain threshold above the peripheral nerve trunk of the arm assess the sensitivity of the nervous tissue to this improper alignment. A recent study identified high pain and lower pressure pain thresholds over the nerve tissue and the upper trapezius and apophyseal joints of the cervical vertebrae during neurodynamic examination in the scapular depression alignment compared with normal scapular alignment without symptoms. This indicates that the subjects with scapular depression alignment had very high mechanical sensitivity to the nerve tissues of the neck and arms. ${ }^{11}$

Neurodynamics is used not only for evaluation but also for treatment. Systematic reviews have emphasized that nerve mobilization is an effective method for improving flexibility, function, pressure pain threshold, and disability and for reducing pain. ${ }^{12}$ However, there is no study yet on mechanical sensitivity after neural mobilization in subjects with scapular depression alignment.

Scapular alignment exercises passively bring the scapula to normal alignment and maintain it with the assistance of the examiner. Therefore, maintaining the scapula in an upward rotational position through alignment exercise is a useful exercise method because it reduces the pressure in the costoclavicular space and reduces the symptoms of thoracic outlet syndrome. ${ }^{13}$ Other researchers have confirmed that the scapular posture alignment strategy elicited the activity of the lower trapezius typing in sitting positions in patients with neck pain. ${ }^{14}$

However, the change in muscle activity and nerve sensitivity after applying the scapular alignment exercise has not yet been evaluated, and studies comparing this exercise with nerve mobilization are insufficient. Thus, this study compared the pressure pain thresholds of the upper trapezius and median nerve trunks and the muscle activity during shoulder flexion before and after scapular alignment exercises and nerve mobilization in subjects with scapular depression alignment.

\section{METHODS}

\section{Participants}

The subjects were 30 men with scapular depression alignment aged 20-40 years old. Necessary sample size was calculated a priori for a power of 0.80 , and effect size of 0.80 , and alpha level of 0.05 by $\mathrm{G}^{*}$ Power software (version 3.1.2). This calculation indicated that the necessary sample size was 14 subjects for the study. The scapular depression alignment was defined as an alignment in which the superior angle of the scapula and the lateral board of the acromion are located under the $2^{\text {nd }}$ spinous process of thoracic spine. ${ }^{5}$ Thirty subjects were randomly divided into the nerve mobilization group $(n=15)$ and the scapular alignment exercise group by coin toss. All subjects were recruited voluntarily after receiving sufficient explanation about the purpose and experimental procedure of this study prior to participation and written informed consent was obtained from all participants. Those who had an orthopedic or neurosurgery history of the arm or neck within the previous year were excluded. The general characteristics of the subjects are presented in Table 1.

\section{Procedure}

The static scapular posture evaluation was performed by the examiner in an upright position with both arms as

Table 1. Comparison of subject characteristics

\begin{tabular}{cccc}
\hline Characteristics & SAE group $(\mathrm{N}=15)$ & NM group $(\mathrm{N}=15)$ & $p$ \\
\hline Age (years) & $32.6 \pm 1.55$ & $33.93 \pm 2.28$ & 0.07 \\
Body mass $(\mathrm{kg})$ & $71.93 \pm 5.96$ & $74.67 \pm 9.09$ & 0.34 \\
Height $(\mathrm{cm})$ & $174.27 \pm 3.01$ & $175.27 \pm 4.13$ & 0.46 \\
\hline
\end{tabular}

Data are expressed as mean $\pm \mathrm{SD}$.

SAE, scapular alignment exercise; NM, nerve mobilization. 
comfortable as possible. The examiner marked the superior angle of scapular (SAS), the acromion (A), and spinous process of $2^{\text {nd }}$ thoracic spine (ST2) with a pen. If SAS and A are located below ST2, they were selected as subjects for scapular depression alignment (Figure 1). ${ }^{5}$ Thirty subjects were randomly divided into the nerve mobilization group $(n=15)$ and the scapular alignment exercise group. The subjects in both groups were measured in terms of the pressure pain threshold and muscle activity during shoulder flexion. Then, each group performed scapular alignment exercises and nerve mobilization for $20 \mathrm{~min}$. After the intervention, the pressure pain threshold and muscle activity during shoulder flexion were repeatedly measured.

\section{Pressure pain threshold}

Pressure pain threshold was measured with a commander algometer (JTECH Medical, USA). The algometer probe was applied vertically to the skin at a rate of $1 \mathrm{~kg} / \mathrm{cm}^{2} / \mathrm{s}$, and the measurement was measured three times at each location, with a $30 \mathrm{~s}$ rest time taken between measurements. The test subjects were instructed to speak when pressure was perceived as pain and aimed at the point where they first felt pain and to express the pain without holding back. The

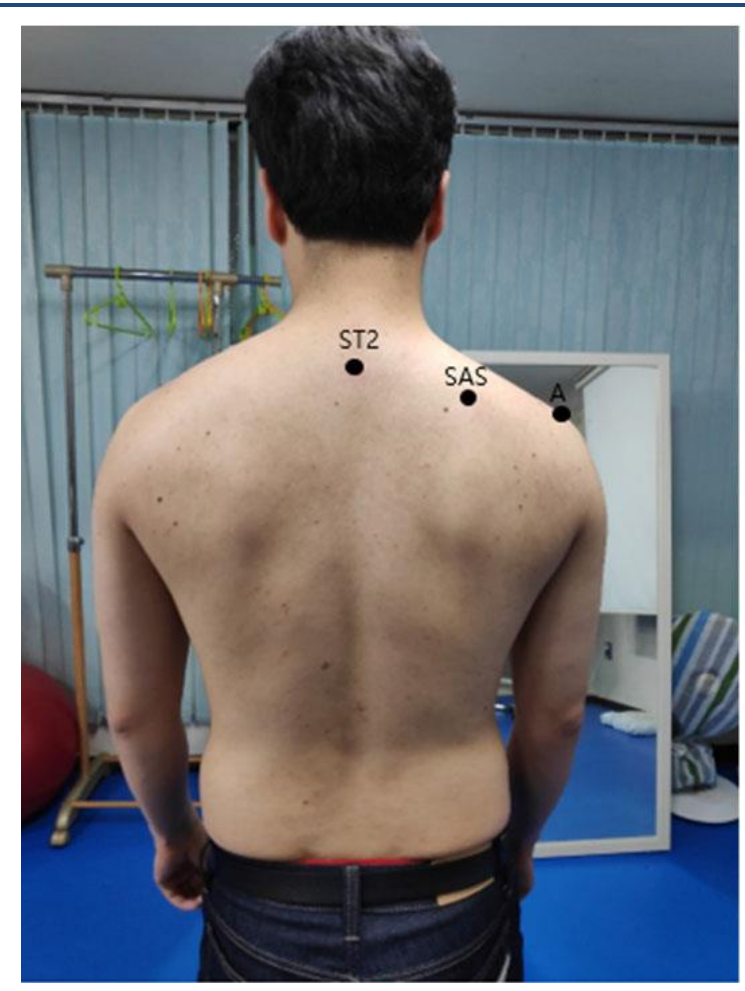

Figure 1. Diagnosis for scapular depression alignment (ST2, spinous process of 2nd thoracic spine; SAS, superior angle of scapular; A, acromion). pressure pain threshold was measured in the median nerve and upper trapezius.

The median nerve was confirmed by palpation at the inner part of the cubital fossa adjacent to the tendon of the biceps brachii muscle in the supine position and the elbow joint extended position ${ }^{15}$. The measurement position of the upper trapezius was taken as the middle point between the lateral board of the acromion and the $\mathrm{C} 7$ spinous process in the sitting position. ${ }^{11}$

\section{Muscle activity}

A wireless surface EMG device (TeleMyo DTS, Noraxon Inc., AZ, USA) was used to measure the muscle activity of the upper trapezius, lower trapezius, and serratus anterior muscles during shoulder flexion. A disposable single-pole surface electrode made of silver and silver chloride was used as the electrode, and the distance between the centers of the electrode was fixed at $2 \mathrm{~cm}$. After obtaining consent from the subjects and exposing the body part to which the electrode would be attached, hair removal was performed using a disposable razor to reduce skin resistance to the signal of the surface EMG. The skin of the attachment site was removed by rubbing with sandpaper 3-5 times. Skin fat was removed with rubbing alcohol.

Electrodes were attached to the muscle belly of the serratus anterior (on the midaxillary line of the right fifth rib), upper trapezius (in the region of the trapezius insertion at the midpoint between the $\mathrm{C} 7$ spinous process and the right acromioclavicular joint), and lower trapezius (position of the diagonal downline approximately $5 \mathrm{~cm}$ from the root of the spine of the scapula). ${ }^{16}$ Shoulder flexion was performed after sitting on a chair with an adjustable height, adjusting the shoulder blade height to the same point, measuring the $120^{\circ}$ flexion, and marking it with a bar to reduce the height deviation between individuals (Figure 2). The collected analog signals of the surface EMG were filtered and processed by a personal computer. The sampling rate of the EMG signal was $1,000 \mathrm{~Hz}$, and the frequency bandwidth was filtered using a $20-400 \mathrm{~Hz}$ band pass filter and a $60 \mathrm{~Hz}$ notch filter to remove noise.

To quantify all collected EMG signals, the root mean square was processed. The collected signals of each muscle were normalized as a percentage (\%maximal voluntary isometric contraction, \%MVIC). To standardize the activity of the muscles, the maximum isometric contraction of each muscle was confirmed in the posture of the manual muscle test. ${ }^{17}$ The average value was used three times for $5 \mathrm{~s}$, and the average number of EMG signals for $3 \mathrm{~s}$ excluding the first and last $1 \mathrm{~s}$ was collected. At least $1 \mathrm{~min}$ of rest was provided between measurements to reduce the likelihood of 


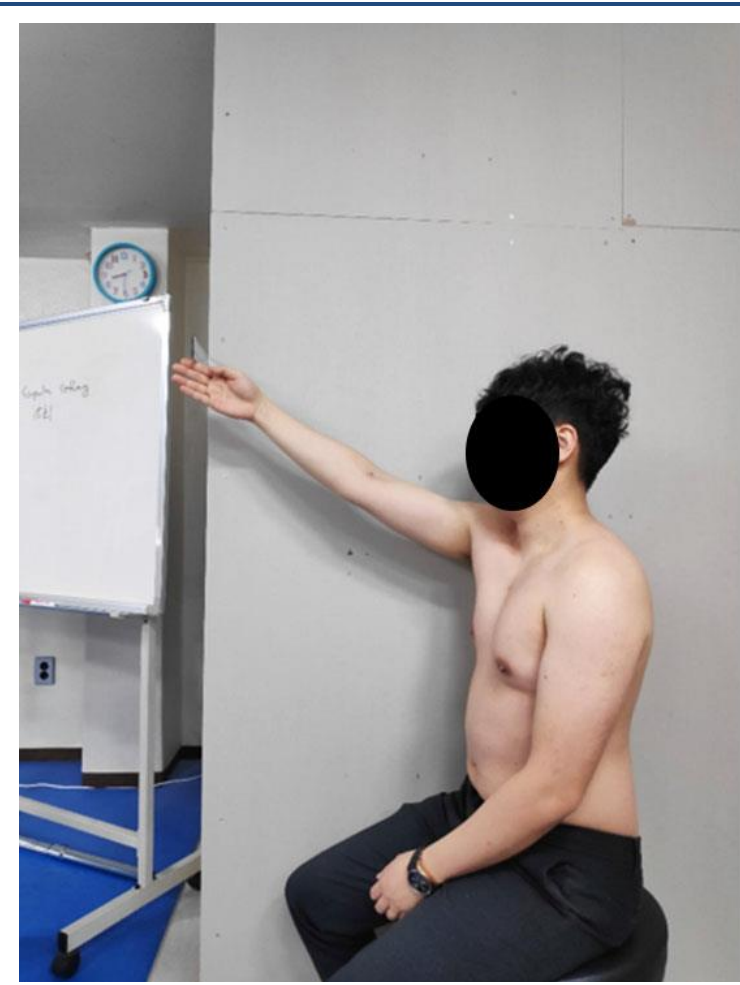

Figure 2. Shoulder flexion for measuring muscle activity.

fatigue.

\section{Nerve mobilization}

Nerve mobilization was performed in the following order: 1) fixation of shoulder girdle, 2) shoulder joint abduction, 3) wrist/finger extension, 4) forearm supination, 5) shoulder joint external rotation, and 6) elbow joint extension. Nerve mobilization was applied using the "sliders" technique, which was found to be safe and has a large sliding range. In nerve mobilization, the elbow joint is extended, the lateral flexion of the neck and head to the ipsilateral side occurs, and the neck and head are lateral flexion to the opposite side when returning to the original position (Figure 3). ${ }^{18}$ Five sets of nerve mobilization were performed, with a $30 \mathrm{~s}$ rest time between sets. In addition, 10 times of nerve mobilizations were performed in the first set, 12 times in the second set, 14 times in the third set, 16 times in the fourth set, and 20 times in the fifth set. The gradual increase in the number of times per set was to minimize the negative reactions to the subject's adaptation threshold and movement. At each frequency, nerve mobilization is performed to the point where the subject reported symptoms or feels resistance by the examiner. After holding it for $1 \mathrm{~s}$ at the end range, it returns to its original position, and the movement is completed for $2 \mathrm{~s}$.

\section{Scapular alignment exercise}

The test subjects stand upright and relax their arms at their sides. The examiner stands next to the subject, holds the subject's medial and lateral board of the scapular with one hand, and gently wraps the acromion and coracoid process in the palm of the other hand. The scapula, which is manually controlled by the examiner, is elevated, slightly rotated upward $\left(10^{\circ}-15^{\circ}\right)$, and posteriorly tilted so that the superior angle of the scapula and the lateral surface of the acromion are parallel to the same height as the spinous process of $2^{\text {nd }}$ thoracic spine.

In addition, the scapula maintains the medial board of the scapula at $7.5 \mathrm{~cm}$ from the spinous process of thoracic spine, and after keeping it close to the thoracic cage so that the medial board of the scapula does not rise, it is rotated $30^{\circ}$ anterior to the frontal plane (Figure 3). ${ }^{5,19,20}$ The examiner instructs the subject to actively maintain this position and
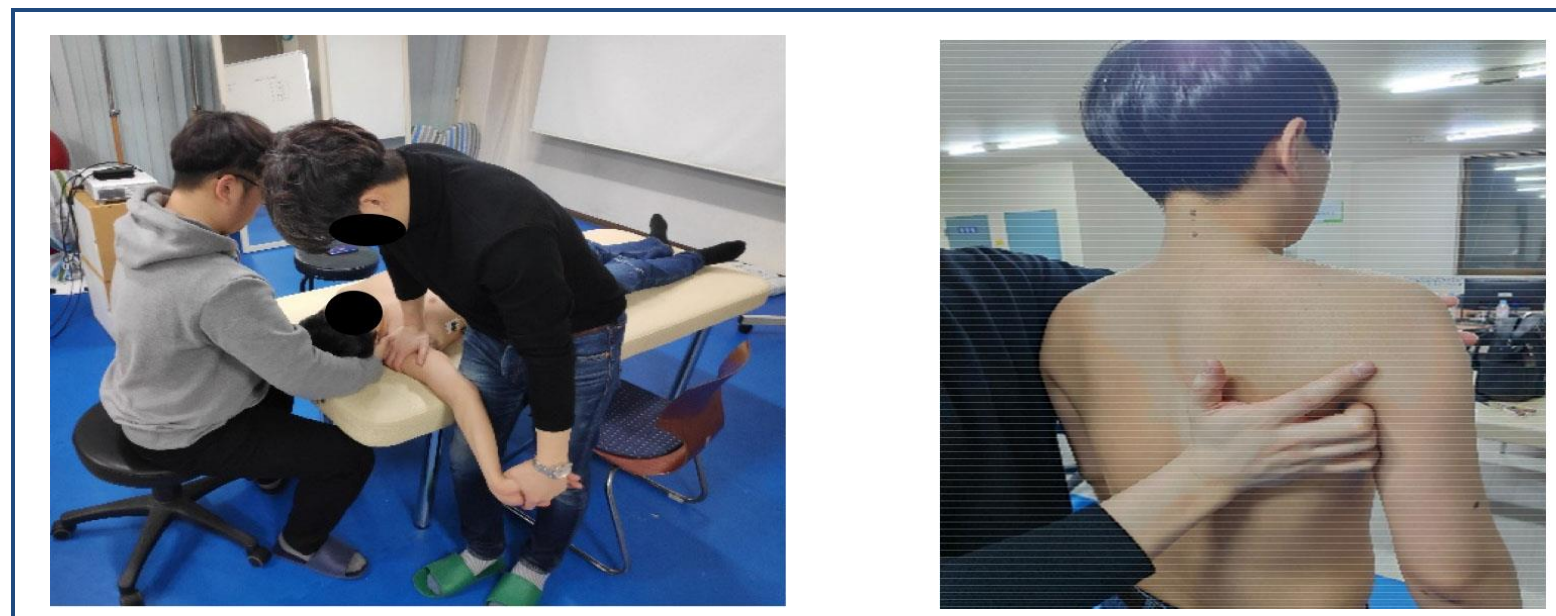

Figure 3. Nerve mobilization (left) and scapular alignment exercise (right). 
confirms that the normal alignment of the scapula is maintained continuously. The application of the exercise was maintained for $1 \mathrm{~min}$ each time for a total of 15 repetitions, and $10 \mathrm{~s}$ of rest was given between repetitions.

\section{Statistical analysis}

The Shapiro-Wilk test was used to test for a normal distribution. A paired $t$-test was performed for the number of changes before and after intervention in both groups, and an independent $t$-test was performed to compare the values of changes between the two groups after intervention. The level of statistical significance was set to $p<0.05$. The Statistical Package for the Social Sciences version 23.0 (SPSS, Inc., Chicago, IL, USA) software was used for the statistical analysis.

\section{RESULTS}

There was a significant increase in the pressure pain threshold of the upper trapezius before and after intervention in the scapular alignment exercise group $(p<0.05)$. No significant difference was found between the two groups according to the intervention $(p>0.05)$ (Table 2$)$. No signifi- cant difference was observed in the pressure pain threshold of median nerve before and after intervention in either group ( $p>0.05$ ) (Table 2).

No significant difference was observed in the muscle activity of the upper and lower trapezius before and after intervention in either group $(p>0.05)$. There was a significant increase in the muscle activity of the serratus anterior before and after intervention in the scapular alignment exercise group $(p<0.05)$, and a significant difference was found in the muscle activity of the serratus anterior between the two groups according to the intervention $(p<0.05)$ (Table 3).

\section{DISCUSSION}

As a result of this study, there was a significant increase in the pressure pain threshold of the upper trapezius in the scapular alignment exercise group after performing the scapular alignment exercise and nerve mobilization, but no significant difference was found between the two groups.

Lee et al. compared the normal scapular alignment group and the scapular depression alignment group and found a significant decrease in the pressure pain threshold in the

Table 2. Comparison for pain pressure threshold (unit: $\mathrm{kg}$ )

\begin{tabular}{ccccccccc}
\hline & & Pre & Post & Change & $t$ & $p$ & $t$ & $p$ \\
\hline Median nerve & SAEG $(\mathrm{n}=15)$ & $4.55 \pm 1.42^{\mathrm{a}}$ & $4.68 \pm 1.17$ & $0.13 \pm 0.83$ & -0.61 & 0.55 & -0.32 & 0.75 \\
& NMG $(\mathrm{n}=15)$ & $4.19 \pm 0.71$ & $4.42 \pm 1.17$ & $0.24 \pm 0.97$ & -0.95 & 0.36 & & \\
Upper trapezius & SAEG $(\mathrm{n}=15)$ & $3.97 \pm 0.98$ & $4.35 \pm 0.83$ & $0.38 \pm 0.47$ & -3.16 & $0.01^{*}$ & \multirow{2}{*}{1.36} & 0.19 \\
& NMG $(\mathrm{n}=15)$ & $3.83 \pm 1.3$ & $3.91 \pm 1.13$ & $0.08 \pm 0.72$ & -0.44 & 0.67 & & \\
\hline
\end{tabular}

SAEG, scapular alignment exercise group; NMG, nerve mobilization group.

${ }^{\mathrm{a}}$ mean $\pm \mathrm{SD}$.

${ }^{*} p<0.05$.

Table 3. Comparison for scapular muscle activity during shoulder flexion (unit: \%MVIC)

\begin{tabular}{clccccccc}
\hline & & Pre & Post & Changes & $t$ & $p$ & $t$ & $p$ \\
\hline $\begin{array}{c}\text { Upper } \\
\text { trapezius }\end{array}$ & SAEG $(\mathrm{n}=15)$ & $15.28 \pm 9.79^{\mathrm{a}}$ & $16.65 \pm 11.82$ & $1.37 \pm 3.58$ & -1.48 & 0.16 & \multirow{2}{*}{0} & 0.98 \\
& NMG $(\mathrm{n}=15)$ & $11.55 \pm 7.14$ & $10.8 \pm 6.87$ & $-0.75 \pm 3.2$ & 0.91 & 0.38 & & \\
Lower & SAEG $(\mathrm{n}=15)$ & $17.21 \pm 8.38$ & $18.36 \pm 9.15$ & $1.15 \pm 2.93$ & -1.52 & 0.15 & 0.97 & 0.34 \\
trapezius & NMG $(\mathrm{n}=15)$ & $18.23 \pm 7.72$ & $18.11 \pm 8.41$ & $-0.12 \pm 4.15$ & 0.11 & 0.91 & & \\
Serratus & SAEG $(\mathrm{n}=15)$ & $33.76 \pm 9.48$ & $38.35 \pm 12.49$ & $4.59 \pm 4.98$ & -3.57 & $0.00^{*}$ & \multirow{2}{*}{3.76} & $0.00^{*}$ \\
anterior & NMG $(\mathrm{n}=15)$ & $31.93 \pm 12.43$ & $30.01 \pm 10.74$ & $1.92 \pm 4.49$ & 1.66 & 0.12 & & \\
\hline
\end{tabular}

SAEG, scapular alignment exercise group; NMG, nerve mobilization group.

${ }^{\mathrm{a}}$ mean \pm SD.

${ }^{*} p<0.05$. 
upper and middle trapezius. ${ }^{6}$ The increase in muscle tissue sensitivity, expressed as a low pressure pain threshold, has been described as a response to the sustained elongation of the upper trapezius muscle maintained in an excessively elongated position due to scapular depression alignment. ${ }^{5}$ Therefore, the scapular alignment exercise can lead to decreased tenderness by reducing the continuous elongation acting on the upper trapezius.

Watson et al. found that the manual correction of the scapula establishes the diagnosis of thoracic outlet syndrome, and that it is a very useful clinical indication of whether the rehabilitation strategy focuses on the strengthening of the scapula stabilizer and whether the change in scapula posture in the movement and resting state would be successful. ${ }^{19}$ If the alignment exercise is maintained for $1 \mathrm{~min}$, traction force can be relieved in the brachial plexus. Therefore, the slight increase in the pressure pain threshold on the median nerve caused by the scapular alignment exercise can be attributed to the decrease in mechanical sensitivity due to the relaxation of the traction and elongation force acting on the peripheral nerve trunk.

The ideal scapular alignment requires the superior angle of the scapula and the lateral board of the acromion to be approximately at the same level as the $2^{\text {nd }}$ thoracic spine without excessive elevation or depression and should be within a $30^{\circ}$ internal rotation with respect to the frontal plane. ${ }^{5}$ Moreover, the medial board of the scapula should be parallel to the spine and located 3 in from the spinous process of the spine. ${ }^{21}$ Compared with the ideal scapular alignment, scapular depression alignment is considered when the superior angle of the scapula is located below the spinous process of the second spine. ${ }^{4}$

There was a significant increase in the muscle activity of the serratus anterior before and after intervention in the scapular alignment exercise group, and a significant difference was found between the two groups according to intervention. Although not significantly different before and after the intervention, the scapular alignment group showed a slight increase in muscle activity in the upper trapezius and lower trapezius compared with the nerve mobilization group. Watson et al. recommended a scapular alignment exercise that maintains the scapula in a neutral position with a slight upward rotation and posterior tilt as needed without excessive elevation or depression. ${ }^{20}$ This exercise is completed by scapula stabilizers, such as the serratus anterior, upper, middle, and lower trapezius. The goal of rehabilitation training is to make the smooth upward rotation of the scapula occur throughout the range of motion through the simultaneous activation of all muscles. The scapular alignment exercise of this study was performed using the method recommended by Watson et al. ${ }^{20}$ As a result, the upper and lower trapezius showed a slight increase, and the serratus anterior showed a significant increase. It is considered that the scapula's elevation, upward rotation, and posterior tilt caused the muscle activation of the upper trapezius, lower trapezius, and serratus anterior.

The muscles in the prolonged elongated position experience over-stretch weakness, and a mild form of strain occurs in the state in which eccentric contraction is not performed against the load ${ }^{4}$. If this is not corrected immediately after a change in muscle length occurs, these conditions can develop into painful muscle strains. Therefore, it is important to improve muscle performance by shortening the muscle to a more normal length as a strategy for correction ${ }^{4}$. The scapular alignment exercise improves muscle performance by keeping the stretched muscles at a more ideal length, thus causing a normal movement of the scapula and shoulder joints.

Lee et al. compared the muscle latency time of the upper and middle trapezius during shrug movement in the scapular depression alignment group and the normal alignment group and found a significant increase in the upper trapezius. ${ }^{6}$ The delayed muscle contraction timing in the scapular depression alignment group was the result of the adaptation of the upper trapezius to other supply/recruitment models to maintain the stability of shrug motion. Moreover, small changes in the underlying posture during movement alter the overall pattern of muscle recruitment. Moreover, if the muscle is in a constantly elongated position, cross-bridge structures in the skeletal muscle that are compressed or stretched become limited and affected by the ability to produce force, and the rate of muscle contraction is primarily limited by the rate of cross-linking at the sarcomere level. ${ }^{16}$

In this study, the nerve mobilization group did not show any significant change in muscle activity after intervention. Several studies support the results of this study. RomeroMoraleda et al. divided the subjects with delayed-onset muscle soreness into the nerve mobilization group and the foam roller group to compare pain, rectus femoris muscle strength, and muscle activity, and both groups were found to have reduced pain. ${ }^{22}$ However, only the foam roller group showed an increase in muscle strength and activity of the rectus femoris. In healthy people, a change in grip strength was observed by applying nerve mobilization and general stretching, and there was no significant change between the two groups. The researchers argued that nerve mobilization applied to healthy subjects was not an effective method for increasing grip strength. ${ }^{23}$ Therefore, nerve mobilization did not cause immediate change in muscle performance, and the change in muscle activity of the muscles around the scapula 
was considered to be achieved by scapular alignment exercise.

This study has some limitations. First, when determining the scapular depression alignment, we did not distinguish between the anterior tilt and the downward rotation of the scapula. Second, time, intervention period, and number of repetitions may be insufficient for intervention methods to correct scapular depression alignment to immediately affect the study results. In future studies, the alignment between scapula depression, anterior tilt, and downward rotation should be distinguished, and not only scapular alignment exercise and nerve mobilization should be considered but also an intervention method combining scapular alignment exercise and nerve mobilization in people with pain.

\section{CONCLUSIONS}

The scapular alignment exercises enable subjects with scapular depression alignment to reduce the mechanical sensitivity of muscles and nervous tissues and bring immediate activation in muscle activity of serratus anterior. This study recommend that scapular alignment exercise could help scapular depression syndrome as a therapeutic exercise method in clinical field.

\section{Key Points}

Question Do scapular alignment exercises and nerve mobilization have an immediate effect on pain and muscle activity in subjects with scapular depression alignment?

Findings There was a significant increase in the pressure pain threshold of the upper trapezius and the muscle activity of the serratus anterior before and after intervention in the scapular alignment exercise group.

Meaning The scapular alignment exercises enable subjects with scapular depression alignment to increase pressure pain threshold of the upper trapezius and activate the serratus anterior muscle.

\section{Article information}

Conflict of Interest Disclosures: None.

Funding/Support: None.

Acknowledgment: None.

Ethic Approval: None.

\section{REFERENCES}

1. Kibler WB, Ludewig PM, McClure PW, et al. Clinical implications of scapular dyskinesis in shoulder injury: the 2013 consensus statement from the 'Scapular Summit'. Br J Sports Med. 2013;47(14):877-885.

2. Burkhart SS, Morgan CD, Kibler WB. The disabled throwing shoulder: spectrum of pathology Part I: pathoanatomy and biomechanics. Arthroscopy. 2003;19(4): 404-420.

3. Cagnie B, Struyf F, Cools A, et al. The relevance of scapular dysfunction in neck pain: a brief commentary. $J$ Orthop Sports Phys Ther. 2014;44(6):435-439.

4. Sahrmann SA. Diagnosis and treatment of movement impairment syndromes. St Louis, MO: Mosby Inc; 2002.

5. Azevedo DC, de Lima Pires T, de Souza Andrade F, et al. Influence of scapular position on the pressure pain threshold of the upper trapezius muscle region. Eur $J$ Pain. 2008;12(2):226-232.

6. Lee KT, Chuang CC, Lai CH, et al. Study of the trapezius muscle region pressure pain threshold and latency time in young people with and without depressed scapula. Man Ther. 2015;20(1):124-129.

7. Helgadottir H, Kristjansson E, Mottram S, et al. Altered alignment of the shoulder girdle and cervical spine in patients with insidious onset neck pain and whiplashassociated disorder. J Appl Biomech. 2011;27(3):181-191.

8. Yaxley GA, Jull GA. A modified upper limb tension test: an investigation of responses in normal subjects. Aust $J$ Physiother. 1991;37(3):143-152.

9. Byl C, Puttlitz C, Byl N, et al. Strain in the median and ulnar nerves during upper-extremity positioning. J Hand Surg Am. 2002;27(6):1032-1040.

10. Kitamura T, Takagi K, Yamaga M, et al. Brachial plexus stretching injuries: microcirculation of the brachial plexus. J Shoulder Elbow Surg. 1995;4(2):118-123.

11. Martinez-Merinero P, Lluch E, Gallezo-Izquierdo T, et al. The influence of a depressed scapular alignment on upper limb neural tissue mechanosensitivity and local pressure pain sensitivity. Musculoskelet Sci Pract. 2017; 29:60-65.

12. Neto T, Freitas SR, Marques M, et al. Effects of lower body quadrant neural mobilization in healthy and low back pain populations: a systematic review and metaanalysis. Musculoskelet Sci Pract. 2017;27:14-22.

13. Larsen K. Postural cues for scapular retraction and depression promote costoclavicular space compression and thoracic outlet syndrome. Anaesthesia, Pain and Intensive Care. 2018;22(2):256-257.

14. Wegner S, Jull G, O'Leary S, et al. The effect of a scapular postural correction strategy on trapezius activity in patients with neck pain. Man Ther. 2010;15(6):562566.

15. Schmid AB, Brunner F, Luomajoki H, et al. Reliability 
of clinical tests to evaluate nerve function and mechanosensitivity of the upper limb peripheral nervous system. BMC Musculoskelet Disord. 2009;10:11.

16. Cram JR, Kasman GS, Holtz J. Introduction to surface electromyography. NY: Aspen Publishers; 1998.

17. Kendall FP, McCreary E, Provance P, et al. Muscles: testing and function, with posture and pain (Kendall, Muscles). Philadelphia: Lippincott Williams \& Wilkins; 2005.

18. Santana HHS, Fernandes de Oliveir IAV, Medrado AP, et al. Neurodynamic mobilization and peripheral nerve regeneration: a narrative review. Inter J Neurorehabilitation. 2015;2(2):163-169.

19. Watson LA, Pizzari T, Balster S. Thoracic outlet syndrome part 1: clinical manifestations, differentiation and treatment pathways. Man Ther. 2009;14(6):586-595.
20. Watson LA, Pizzari T, Balster S. Thoracic outlet syndrome part 2: conservative management of thoracic outlet. Man Ther. 2010;15(4):305-314.

21. Sobush DC, Simoneau GG, Dietz KE, et al. The lennie test for measuring scapular position in healthy young adult females: a reliability and validity study. J Orthop Sports Phys Ther. 1996;23(1):39-50.

22. Romero-Moraleda B, La Touche R, Lerma-Lara S, et al. Neurodynamic mobilization and foam rolling improved delayed-onset muscle soreness in a healthy adult population: a randomized controlled clinical trial. PeerJ. 2017;5:e3908-3925.

23. Araujo BF, Nascimento $\mathrm{CMd}$, Busarello FdO, et al. Assessment of handgrip strength after neural mobilization. Rev Bras Med Esporte. 2012;18:242-245. 